\title{
Positive solutions to boundary value problems of a high-order fractional differential equation in a Banach space
}

Yulin Zhao*, Lianjiao Yang and Pingge Chen

"Correspondence: zhaoylch@sina.com School of Science, Hunan University of Technology, Zhuzhou, 412007, China

\begin{abstract}
In this paper, by using the fixed-point theorem in the cone of strict-set-contraction operators, we study a class of higher-order boundary value problems of nonlinear fractional differential equation in a Banach space. The sufficient conditions for the existence of at least two positive solutions is obtained. In addition, an example to illustrate the main results is given.
\end{abstract}

Keywords: fractional differential equation; boundary value problem; measure of noncompactness; strict-set-contraction operators

\section{Introduction}

Fractional calculus is a generalization of ordinary differentiation and integration to arbitrary noninteger order. The fractional differential equations have been of great interest recently. This is because of both the intensive development of the theory of fractional calculus itself and its numerous applications in various fields of science and engineering including fluid flow, rheology, control, electrochemistry, electromagnetic, porous media and probability, etc. (see [1-4]).

In recent years, the existence and uniqueness of solutions of the initial and boundary value problems for fractional equations have been extensively studied (see [4-16] and the references therein). But there are few works that deal with the existence of solutions of nonlinear fractional differential equations in Banach spaces; see [17-24]. In [19], Hussein investigated the existence of pseudo solutions for the following nonlinear $m$-point boundary value problem of fractional type:

$$
\left\{\begin{array}{l}
D_{0^{+}}^{q} u(t)+a(t) f(t, u(t))=0, \quad 0<t<1, \\
u(0)=u^{\prime}(0)=\cdots=u^{(n-2)}(0)=0, \quad u(1)=\sum_{i=1}^{m-2} \zeta_{i} u\left(\eta_{i}\right)
\end{array}\right.
$$

in a reflexive Banach space $E$, where $D_{0^{+}}^{q}$ is the pseudo fractional differential operator of order $n-1<q \leq n, n \geq 2$.

In [22], by the monotone iterative technique and the Mönch fixed point theorem, Lv et $a l$. investigated the existence of a solution to the following Cauchy problem for the differ-

\section{Springer}

C2013 Zhao et al.; licensee Springer. This is an Open Access article distributed under the terms of the Creative Commons Attribution License (http://creativecommons.org/licenses/by/2.0), which permits unrestricted use, distribution, and reproduction in any medium, provided the original work is properly cited. 
ential equation with fractional order in a real Banach space $E$ :

$$
{ }^{C} D^{q} u(t)=f(t, u(t)), \quad u(0)=u_{0},
$$

where ${ }^{C} D^{q} u(t)$ is the Caputo derivative of order $0<q<1$.

By means of Darbo's fixed point theorem, Su [23] established the existence result of solutions to the following boundary value problem of fractional differential equation on unbounded domain $[0,+\infty)$ :

$$
\left\{\begin{array}{l}
D_{0^{+}}^{q} u(t)=f(t, u(t)), \quad t \in[0,+\infty), 1<q \leq 2, \\
u(0)=\theta, \quad D_{0^{+}}^{q-1} u(\infty)=u_{\infty}
\end{array}\right.
$$

in a Banach space $E . D_{0^{+}}^{q}$ is the Riemann-Liouville fractional derivative.

Being directly inspired by $[9,15,19,22]$ but taking quite a different method from that in $[15,19-23]$, we discuss in this paper the following high-order boundary value problem (BVP for short) in a Banach space $E$ :

$$
\left\{\begin{array}{l}
D_{0+}^{q} u(t)+f\left(t, u, u^{\prime}, \ldots, u^{(n-2)}\right)=\theta, \quad \forall t \in(0,1), q \in(n-1, n] \\
u^{(i)}(0)=0, \quad 0 \leq i \leq n-3, \\
\alpha u^{(n-2)}(0)-\beta u^{(n-1)}(0)=\theta, \quad \gamma u^{(n-2)}(1)+\delta u^{(n-1)}(1)=\theta
\end{array}\right.
$$

where $\theta$ is the zero element of $E, n \geq 2, \alpha, \beta, \gamma$ and $\delta$ are nonnegative constants satisfying $\rho^{-1}=\alpha \gamma+\alpha \delta+\beta \gamma>0$, and $D_{0+}^{q}$ is the Caputo fractional derivative. Note that the nonlinear term $f$ depends on $u$ and its derivatives $u^{\prime}, u^{\prime \prime}, \ldots, u^{(n-2)}$.

The paper is organized as follows. In Section 2 we give some basic definitions in Riemann-Liouville fractional calculus and the Kuratowski noncompactness. In Section 3 we present the expression and properties of Green's function associated with BVP (1.1), and by using the fixed-point theorem for strict-set-contraction operators and introducing a new cone $\Omega$, we obtain the existence of at least two positive solutions for BVP (1.1) under certain conditions on the nonlinearity. Moreover, an example illustrating our main result is given in Section 4.

\section{Preliminaries and lemmas}

For convenience of the reader, we present here some definitions and preliminaries which are used throughout the paper. These definitions and lemmas can be found in the recent literature such as $[1,5]$.

Definition 2.1 ([1]) The Riemann-Liouville fractional integral of order $q>0$ of a function $y(t)$ is given by

$$
I_{0+}^{q} y(t)=\frac{1}{\Gamma(q)} \int_{0}^{t}(t-s)^{q-1} y(s) d s
$$

provided that the right-hand side is defined pointwise. 
Definition 2.2 ([1]) The fractional derivative of order $q>0$ of a function $y: \mathbf{R}_{0}^{+} \rightarrow \mathbf{R}$ is given by

$$
D_{0+}^{q} y(t)=\frac{1}{\Gamma(n-q)}\left(\frac{d}{d t}\right)^{n} \int_{0}^{t}(t-s)^{n-q-1} y(s) d s,
$$

where $n=[q]+1,[q]$ denotes the integer part of number $q$, provided that the right-hand side is defined pointwise. In particular, for $q=n, D_{0+}^{q} y(t)=y^{(n)}(t)$.

Lemma 2.3 ([1]) Let $q>0$. Then the fractional differential equation

$$
D_{0+}^{q} y(t)=0
$$

has the unique solution $y(t)=c_{1} t^{q-1}+c_{2} t^{q-2}+\cdots+c_{n} t^{q-n}, c_{i} \in \mathbf{R}, i=1,2, \ldots, n$, here $n-1<$ $q \leq n$.

Lemma 2.4 ([5]) Let $q>0$. Then the following equality holds for $y \in L(0,1), D_{0+}^{q} y \in L(0,1)$ :

$$
I_{0+}^{q} D_{0+}^{q} y(t)=y(t)+c_{1} t^{q-1}+c_{2} t^{q-2}+\cdots+c_{N} t^{q-N}
$$

for some $c_{i} \in \mathbf{R}, i=1,2, \ldots, N$, here $N$ is the smallest integer greater than or equal to $q$.

Let the real Banach space $E$ with the norm $\|\cdot\|$ be partially ordered by a cone $P$ of $E$, i.e., $u \leq v$ if and only if $v-u \in P$, and $P$ is said to be normal if there exists a positive constant $N$ such that $\theta \leq u \leq v$ implies $\|u\| \leq N\|v\|$, where the smallest $N$ is called the normal constant of $P$. For details on cone theory, see [25].

The basic space used in this paper is $C[I, E]$. For any $u \in C[I, E]$, evidently, $\left(C[I, E],\|\cdot\|_{C}\right)$ is a Banach space with the norm $\|u\|_{C}=\sup _{t \in I}|u(t)|$, and $P=\{u \in C[I, E]: u(t) \geq \theta$ for $t \in$ $I\}$ is a cone of the Banach space $C[I, E]$. We use $\alpha, \alpha_{C}$ to denote the Kuratowski noncompactness measure of bounded sets in the spaces $E, C(I, E)$, respectively. As for the definition of the Kuratowski noncompactness measure, we refer to Ref. [25].

Definition 2.5 ([25], Strict-set contraction operator) Let $E_{1}, E_{2}$ be real Banach spaces, $S \subset E_{1} . T: S \rightarrow E_{2}$ is a continuous and bounded operator. If there exists a constant $k$ such that $\alpha(T(S)) \leq k \alpha(S)$, then $T$ is called a $k$-set contraction operator. When $k<1, T$ is called a strict-set contraction operator.

Lemma 2.6 ([25]) If $D \subset C[I, E]$ is bounded and equicontinuous, then $\alpha(D(t))$ is continuous on I and

$$
\alpha_{C}(D)=\max _{t \in I} \alpha(D(t)), \quad \alpha\left(\left\{\int_{I} u(t) d t: u \in D\right\}\right) \leq \int_{I} \alpha(D(t)) d t
$$

where $D(t)=\{u(t): u \in D, t \in I\}$.

Lemma 2.7 ([25]) Let $K$ be a cone in a Banach space E. Assume that $\Omega_{1}, \Omega_{2}$ are open subsets of $E$ with $\theta \in \Omega_{1}, \bar{\Omega}_{1} \subset \Omega_{2}$. If $T: K \cap\left(\bar{\Omega}_{2} \backslash \Omega_{1}\right) \rightarrow K$ is a strict-set contraction operator such that either 
(i) $\|T x\| \leq\|x\|, x \in K \cap \partial \Omega_{1}$ and $\|T x\| \geq\|x\|, x \in K \cap \partial \Omega_{2}$, or

(ii) $\|T x\| \geq\|x\|, x \in K \cap \partial \Omega_{1}$ and $\|T x\| \leq\|x\|, x \in K \cap \partial \Omega_{2}$,

then $T$ has a fixed point in $K \cap\left(\bar{\Omega}_{2} \backslash \Omega_{1}\right)$.

In the paper, we always assume that the following three assumptions hold:

(H0) $0<\rho^{*}:=\rho \int_{0}^{1} \varphi(s) d s<+\infty$, where $\varphi(s)$ is defined as

$$
\varphi(s)=\frac{1}{\Gamma(q-n+2)}(\beta+\alpha s)[\gamma(1-s)+\delta(q-n+1)](1-s)^{q-n} .
$$

(H1) There exist $a \in C\left[I, R_{+}\right]$and $h \in C\left[R_{+}^{n-1}, R_{+}\right]$such that

$$
\begin{gathered}
\left\|f\left(t, u_{1}, \ldots, u_{n-1}\right)\right\| \leq a(t) h\left(\left\|u_{1}\right\|, \ldots,\left\|u_{n-1}\right\|\right), \\
\forall t \in I, u_{k} \in P, k=1, \ldots, n-1 .
\end{gathered}
$$

(H2) $f: I \times P_{r}^{n-1} \rightarrow P$ for any $r>0, f$ is uniformly continuous on $I \times P_{r}^{n-1}$ and there exist nonnegative constants $L_{k}, k=1, \ldots, n-1$, with

$$
\frac{2}{\rho^{*}}\left(\sum_{k=1}^{n-2} \frac{L_{k}}{(n-2-k) !}+L_{n-1}\right)<1
$$

such that

$$
\alpha\left(f\left(t, D_{1}, D_{2}, \ldots, D_{n-1}\right)\right) \leq \sum_{k=1}^{n-1} L_{k} \alpha\left(D_{k}\right), \quad \forall t \in I, \text { bounded } D_{k} \in P_{r},
$$

where $P_{r}=\{u \in P:\|u\| \leq r\}$.

\section{Main results}

Lemma 3.1 Given $y \in C[I, E]$, then the unique solution of

$$
\begin{cases}D_{0^{+}}^{q-n+2} x(t)+y(t)=0, & 0<t<1, n-1<q \leq n, n>2, \\ \alpha x(0)-\beta x^{\prime}(0)=0, & \gamma x(1)+\delta x^{\prime}(1)=0\end{cases}
$$

is

$$
x(t)=\int_{0}^{1} G(t, s) y(s) d s
$$

where

$$
G(t, s)= \begin{cases}G_{1}(t, s)=-\frac{(t-s)^{q-n+1}}{\Gamma(q-n+2)}+G_{0}(t, s), & 0 \leq s \leq t \leq 1 \\ G_{0}(t, s), & 0 \leq t \leq s \leq 1\end{cases}
$$

and

$$
G_{0}(t, s)=\frac{\rho(\beta+\alpha t)}{\Gamma(q-n+2)}[\gamma(1-s)+\delta(q-n+1)](1-s)^{q-n} .
$$


Proof Deduced from Lemma 2.4, we have

$$
x(t)=-I_{0+}^{q-n+2} y(t)+c_{1}+c_{2} t
$$

for some $c_{1}, c_{2} \in \mathbf{R}$. Then we get

$$
\begin{aligned}
& x(t)=-\frac{1}{\Gamma(q-n+2)} \int_{0}^{t}(t-s)^{q-n+1} y(s) d s+c_{1}+c_{2} t \\
& x^{\prime}(t)=-\frac{1}{\Gamma(q-n+1)} \int_{0}^{t}(t-s)^{q-n} y(s) d s+c_{2} .
\end{aligned}
$$

By boundary conditions $\alpha x(0)-\beta x^{\prime}(0)=0, \gamma x(1)+\delta x^{\prime}(1)=0$, and noting that $\Gamma(q-n+2)=$ $(q-n+1) \Gamma(q-n+1)$, we have

$$
c_{1}=\frac{\rho \beta}{\Gamma(q-n+2)} \int_{0}^{1}[\gamma(1-s)+\delta(q-n+1)](1-s)^{q-n} y(s) d s
$$

and

$$
c_{2}=\frac{\rho \alpha}{\Gamma(q-n+2)} \int_{0}^{1}[\gamma(1-s)+\delta(q-n+1)](1-s)^{q-n} y(s) d s .
$$

Thus

$$
\begin{aligned}
x(t)= & -\int_{0}^{t} \frac{(t-s)^{q-n+1}}{\Gamma(q-n+2)} y(s) d s \\
& +\frac{\rho \beta}{\Gamma(q-n+2)} \int_{0}^{1}[\gamma(1-s)+\delta(q-n+1)](1-s)^{q-n} y(s) d s \\
& +\frac{\rho \alpha t}{\Gamma(q-n+2)} \int_{0}^{1}[\gamma(1-s)+\delta(q-n+1)](1-s)^{q-n} y(s) d s \\
= & -\int_{0}^{t} \frac{(t-s)^{q-n+1}}{\Gamma(q-n+2)} y(s) d s+\rho(\beta+\alpha t) \int_{0}^{1} \frac{[\gamma(1-s)+\delta(q-n+1)](1-s)^{q-n}}{\Gamma(q-n+2) d s} \\
= & \int_{0}^{1} G(t, s) y(s) d s,
\end{aligned}
$$

where $G(t, s)$ is Green's function defined by (3.3). This completes the proof.

Moreover, there is one paper [10] in which the following statement was shown.

Lemma $3.2([10])$ The function $G(t, s)$ defined by Lemma 2.3 has the following properties:

(i) $G(t, s)$ is continuous on $[0,1] \times[0,1]$;

(ii) if $\beta>\frac{n-q}{q-n+1} \alpha$, then $0<G(t, s) \leq G(s, s)$ for any $t, s \in[0,1]$.

Lemma 3.3 If $\beta>\frac{n-q}{q-n+1} \alpha$, then the function $G(t, s)$ satisfies:

$\lambda \varphi(s) \leq G(t, s) \leq \rho \varphi(s) \quad$ for $t, s \in[0,1]$ 
where

$$
\begin{aligned}
& \lambda:=\min \left\{\frac{4 \rho \alpha \gamma \delta[\beta(q-n+1)+\alpha(q-n)]}{\zeta}, \frac{4 \rho \alpha \beta \gamma \delta(q-n+1)}{\zeta}\right\}, \\
& \zeta:=[\alpha \gamma-\beta \gamma+\alpha \delta(q-n+1)]^{2}+4 \alpha \beta \gamma[\gamma+\delta(q-n+1)] .
\end{aligned}
$$

Proof According to (3.3) and Lemma 3.2, we have

$$
G^{*}(s) \leq G(t, s) \leq \rho \varphi(s),
$$

where

$$
G^{*}(s)= \begin{cases}G_{1}(1, s), & 0 \leq s<\frac{\beta \gamma-\alpha \delta(q-n)}{\alpha \delta+\beta \gamma}, \\ G_{0}(0, s), & \frac{\beta \gamma-\alpha \delta(q-n)}{\alpha \delta+\beta \gamma} \leq s<1 .\end{cases}
$$

Since

$$
\begin{aligned}
\inf _{0<s<1} \frac{G_{1}(1, s)}{\varphi(s)} & =\inf _{0<s<1} \frac{-\rho^{-1}(1-s)+(\beta+\alpha)[\gamma(1-s)+\delta(q-n+1)]}{\rho^{-1}(\beta+\alpha s)[\gamma(1-s)+\delta(q-n+1)]} \\
& \geq \frac{\rho \delta[\beta(q-n+1)+\alpha(q-n)]}{(\beta+\alpha s)[\gamma(1-s)+\delta(q-n+1)]} \\
& \geq \frac{4 \rho \alpha \gamma \delta[\beta(q-n+1)+\alpha(q-n)]}{[\alpha \gamma-\beta \gamma+\alpha \delta(q-n+1)]^{2}+4 \alpha \beta \gamma[\gamma+\delta(q-n+1)]} \\
& =\frac{4 \rho \alpha \gamma \delta[\beta(q-n+1)+\alpha(q-n)]}{\zeta},
\end{aligned}
$$

and

$$
\begin{aligned}
\inf _{0<s<1} \frac{G_{0}(0, s)}{\varphi(s)} & =\inf _{0<s<1} \frac{\rho[\beta \gamma(1-s)+\beta \delta(q-n+1)]}{(\beta+\alpha s)[\gamma(1-s)+\delta(q-n+1)]} \\
& \geq \frac{4 \rho^{-1} \alpha \beta \gamma \delta(q-n+1)}{[\alpha \gamma-\beta \gamma+\alpha \delta(q-n+1)]^{2}+4 \alpha \beta \gamma[\gamma+\delta(q-n+1)]} \\
& =\frac{4 \rho \alpha \beta \gamma \delta(q-n+1)}{\zeta},
\end{aligned}
$$

then we get

$$
\lambda \varphi(s) \leq G(t, s) \leq G(s, s) \leq \rho \varphi(s)
$$

Lemma 3.4 Let $u(t)=I_{0^{+}}^{n-2} x(t), x \in C[I, E]$. Then problem (1.1) can be transformed into the following modified problem:

$$
\left\{\begin{array}{l}
D_{0^{+}}^{q-n+2} x(t)+f\left(s, I_{0^{+}}^{n-2} x(s), \ldots, I_{0^{+}}^{1} x(s), x(s)\right)=\theta, \\
\alpha x(0)-\beta x^{\prime}(0)=\theta, \quad \gamma x(1)+\delta x^{\prime}(1)=\theta,
\end{array}\right.
$$

where $0<t<1, n-1<q \leq n, n \geq 2$. Moreover, if $x \in C[I, E]$ is a solution of problem (3.3) and $x \geq \theta, x \neq \equiv$, then the function $u(t)=I_{0^{+}}^{n-2} x(s)$ is a positive solution of $(1.1)$. 
To obtain a positive solution, we construct a cone $\Omega$ by

$$
\Omega=\left\{x(t) \in P: x(t) \geq \frac{\lambda}{\rho} x(s), \forall t, s \in I\right\}
$$

where $P=\{x \in C[I, E]: x(t) \geq \theta, t \in I\}$.

Define an integral operator $T: \Omega \rightarrow E$ by

$$
(T x)(t)=\int_{0}^{1} G(t, s) f\left(s, I_{0^{+}}^{n-2} x(s), I_{0^{+}}^{n-3} x(s), \ldots, I_{0^{+}}^{1} x(s), x(s)\right) d s, \quad 0 \leq t \leq 1 .
$$

Lemma 3.5 Assume that (H0)-(H2) hold. Then $T: \Omega \rightarrow \Omega$ is a strict-set contraction operator.

Proof From Lemma 3.3 and (3.6), we obtain

$$
(T x)(t) \geq \lambda \int_{0}^{1} \varphi(s) f\left(s, I_{0^{+}}^{n-2} x_{j}(s), \ldots, I_{0^{+}}^{1} x_{j}(s), x_{j}(s)\right) d s
$$

On the other hand,

$$
\begin{aligned}
(T x)(s) & =\int_{0}^{1} G(s, s) f\left(s, I_{0^{+}}^{n-2} x_{j}(s), \ldots, I_{0^{+}}^{1} x_{j}(s), x_{j}(s)\right) d s \\
& \leq \rho \int_{0}^{1} \varphi(s) f\left(s, I_{0^{+}}^{n-2} x_{j}(s), \ldots, I_{0^{+}}^{1} x_{j}(s), x_{j}(s)\right) d s \\
& =\frac{\rho}{\lambda} \int_{0}^{1} \lambda \varphi(s) f\left(s, I_{0^{+}}^{n-2} x_{j}(s), \ldots, I_{0^{+}}^{1} x_{j}(s), x_{j}(s)\right) d s \leq \frac{\rho}{\lambda}(T x)(t) .
\end{aligned}
$$

Then $(T x)(t) \geq \frac{\lambda}{\rho}(T x)(s)$, which implies $(T x) \in \Omega$, i.e., $T(\Omega) \subset \Omega$.

Next we prove that $T$ is continuous on $\Omega$. Let $\left\{x_{j}\right\},\{x\} \subset \Omega$ and $\left\|x_{j}-x\right\|_{\Omega} \rightarrow 0(j \rightarrow \infty)$. Hence $\left\{x_{j}\right\}$ is a bounded subset of $\Omega$. Thus, there exists $r>0$ such that $r=\sup _{j}\left\|x_{j}\right\|_{\Omega}<\infty$ and $\|x\|_{\Omega} \leq r$.

According to the properties of $f$, for $\forall \varepsilon>0$, there exists $J>0$ such that

$$
\left\|f\left(s, I_{0^{+}}^{n-2} x_{j}(s), \ldots, I_{0^{+}}^{1} x_{j}(s), x_{j}(s)\right)-f\left(s, I_{0^{+}}^{n-2} x(s), \ldots, I_{0^{+}}^{1} x(s), x(s)\right)\right\| \leq \frac{\varepsilon}{\rho^{*}}
$$

for $j \geq J, \forall t \in I$.

Then

$$
\begin{aligned}
\left\|\left(T x_{j}\right)(t)-(T x)(t)\right\| \leq & \int_{0}^{1} G(t, s) \| f\left(s, I_{0^{+}}^{n-2} x_{j}(s), \ldots, I_{0^{+}}^{1} x_{j}(s), x_{j}(s)\right) \\
& -f\left(s, I_{0^{+}}^{n-2} x(s), \ldots, I_{0^{+}}^{1} x(s), x(s)\right) \| d s \\
\leq & \rho \int_{0}^{1} \varphi(s) d s \cdot \| f\left(s, I_{0^{+}}^{n-2} x_{j}(s), \ldots, I_{0^{+}}^{1} x_{j}(s), x_{j}(s)\right) \\
& -f\left(s, I_{0^{+}}^{n-2} x(s), \ldots, I_{0^{+}}^{1} x(s), x(s)\right) \|<\varepsilon .
\end{aligned}
$$


Therefore, $\forall \varepsilon>0$ for any $t \in I$ and $j \geq J$. We get

$$
\left\|\left(T x_{j}\right)(t)-(T x)(t)\right\| \rightarrow 0
$$

This implies $T$ is continuous on $\Omega$.

By the properties of continuity of $G(t, s)$, it is easy to see that $T$ is equicontinuous on $I$.

Finally, we are going to show that $T$ is a strict-set contraction operator. Let $D \subset \Omega$ be bounded. Then, by condition (H1), Lemma 3.1 implies $\alpha_{C}(T D)=\max _{t \in I} \alpha((T D)(t))$. It follows from (3.6) that

$$
\begin{aligned}
\alpha((T D)(t)) & =\alpha\left(\left\{\int_{0}^{1} G(t, s) f\left(s,\left(T_{1} x\right)(s), \ldots,\left(T_{n-2} x\right)(s), x(s)\right) d s \in D\right\}\right) \\
& \leq \int_{0}^{1} \alpha\left(\left\{G(t, s) f\left(s,\left(T_{1} x\right)(s), \ldots,\left(T_{n-2} x\right)(s), x(s)\right) d s \in D\right\}\right) \\
& \leq \rho \int_{0}^{1} \varphi(s) \alpha\left(f\left(I \times\left(I_{0^{+}}^{n-2} D\right)(I) \times \cdots \times\left(I_{0^{+}}^{1} D\right)(I) \times D(I)\right)\right) d s \\
& \leq \rho^{*}\left(\sum_{k=1}^{n-2} L_{k} \alpha\left(\left(I_{0^{+}}^{n-1-k} D\right)(I)\right)+L_{n-1} \alpha(D(I))\right),
\end{aligned}
$$

which implies

$$
\alpha_{C}(T D) \leq \rho^{*}\left(\sum_{k=1}^{n-2} L_{k} \alpha\left(\left(I_{0^{+}}^{n-1-k} D\right)(I)\right)+L_{n-1} \alpha(D(I))\right) .
$$

Obviously,

$$
\begin{aligned}
\alpha\left(I_{0^{+}}^{n-1-k} D\right)(I) & =\alpha\left(\left\{\int_{0}^{s} \frac{(s-v)^{n-2-k}}{(n-2-k) !} x(s) d s: v \in[0, s], s \in I, k=1, \ldots, n-2\right\}\right) \\
& \leq \frac{1}{(n-2-k) !} \alpha(D(I)) .
\end{aligned}
$$

Using a similar method as in the proof of Theorem 2.1.1 in [25], we have

$$
\alpha(D(I)) \leq 2 \alpha_{C}(D)
$$

Therefore, it follows from (3.7), (3.8) and (3.9) that

$$
\alpha_{C}(T D) \leq \frac{2}{\rho^{*}}\left(\sum_{k=1}^{n-2} \frac{L_{k}}{(n-2-k) !}+L_{n-1}\right) \alpha_{C}(D)
$$

Noticing that (2.3), we obtain that $T$ is a strict-set contraction operator. This completes the proof.

Now we are in a position to give the main result of this work.

Theorem 3.6 Let the cone P be normal and conditions (H0)-(H2) hold. In addition, assume that the following conditions are satisfied: 
(H3) There exist $u^{*} \in P \backslash\{\theta\}, c_{1} \in C\left[I, R_{+}\right]$and $h_{1} \in C\left[P^{n-1}, R_{+}\right]$such that

$$
f\left(t, u_{1}, \ldots, u_{n-1}\right) \geq c_{1}(t) h_{1}\left(u_{1}, \ldots, u_{n-1}\right) u^{*}, \quad \forall t \in I, u_{k} \in P
$$

and

$$
h_{1}^{\infty}=\lim _{\sum_{k=1}^{n-1}\left\|u_{k}\right\| \rightarrow \infty} \frac{h_{1}\left(u_{1}, \ldots, u_{n-1}\right)}{\sum_{k=1}^{n-1}\left\|u_{k}\right\|}>\triangle^{-1}, \quad \text { as } u_{k} \in P
$$

where $\triangle:=N^{-2} \rho^{-1}\left(\lambda^{2} \int_{0}^{1} \varphi(s) c_{1}(s) d s\left\|u^{*}\right\|\right)$.

(H4) There exist $u_{*} \in P \backslash\{\theta\}, c_{2} \in C\left[I, R_{+}\right]$and $h_{2} \in C\left[P^{n-1}, R_{+}\right]$such that

$$
f\left(t, u_{1}, \ldots, u_{n-2}\right) \geq c_{2}(t) h_{2}\left(u_{1}, \ldots, u_{n-1}\right) u_{*}, \quad \forall t \in I, u_{k} \in P,
$$

and

$$
h_{1}^{0}=\lim _{\sum_{k=1}^{n-1}\left\|u_{k}\right\| \rightarrow 0} \frac{h_{2}\left(u_{1}, \ldots, u_{n-1}\right)}{\sum_{k=1}^{n-1}\left\|u_{i}\right\|}>\nabla^{-1}, \quad \text { as } u_{k} \in P \text {, }
$$

where $\nabla:=N^{-2} \rho^{-1}\left(\lambda^{2} \int_{0}^{1} \varphi(s) c_{2}(s) d s\left\|u_{*}\right\|\right)$.

(H5) There exists $\xi>0$ such that

$$
\rho N M_{\xi} \int_{0}^{1} \varphi(s) a(s) d s<\xi
$$

where $M_{\xi}=\max _{u_{k} \in P_{\xi}}\left\{h\left(\left\|u_{1}\right\|, \ldots,\left\|u_{n-1}\right\|\right)\right\}$

Then problem (1.1) has at least two different positive solutions.

Proof Consider condition (H3), there exists $r_{1}>0$ such that

$$
h_{1}\left(u_{1}, \ldots, u_{n-1}\right) \geq\left(h_{1}^{\infty}-\varepsilon_{1}\right) \sum_{k=1}^{n-1}\left\|u_{k}\right\|, \quad \forall u_{k} \in P, \sum_{k=1}^{n-1}\left\|u_{k}\right\| \geq r_{1}
$$

where $\varepsilon_{1}>0$ satisfies $\left(h_{1}^{\infty}-\varepsilon_{1}\right) \triangle \geq 1$.

Therefore,

$$
f\left(t, u_{1}, \ldots, u_{n-1}\right) \geq\left(h_{1}^{\infty}-\varepsilon_{1}\right) \sum_{k=1}^{n-1}\left\|u_{k}\right\| \cdot c_{1}(t) u^{*}, \quad \forall u_{k} \in P, \sum_{k=1}^{n-1}\left\|u_{k}\right\| \geq r_{1}
$$

Take

$$
R>\max \left\{N \rho \lambda^{-1} r_{1}, \xi\right\}
$$

Then, for $t \in I, x \in \Omega,\|x\|_{\Omega}=R$, we have by (3.5)

$$
\|x(t)\| \geq \lambda(\rho N)^{-1}\|x\|_{\Omega} \geq \lambda(\rho N)^{-1} R>r_{1} .
$$


Thus

$$
\begin{aligned}
(T x)(t) & =\int_{0}^{1} G(t, s) f\left(s, I_{0^{+}}^{n-2} x(s), \ldots, I_{0^{+}}^{1} x(s), x(s)\right) d s \\
& \geq \int_{0}^{1} \lambda \varphi(s) f\left(s, I_{0^{+}}^{n-2} x(s), \ldots, I_{0^{+}}^{1} x(s), x(s)\right) d s \\
& \geq\left(h_{1}^{\infty}-\varepsilon_{1}\right) \lambda \int_{0} u^{1} \varphi(s)\left(\sum_{k=1}^{n-2}\left\|I_{0^{+}}^{n-1-k} x(s)\right\|+\|x(s)\|\right) c_{1}(s) d s \cdot u^{*} \\
& \geq\left(h_{1}^{\infty}-\varepsilon_{1}\right) \lambda \int_{0}^{1} \varphi(s) c_{1}(s)\|x(s)\| d s \cdot u^{*} \\
& \geq\left(h_{1}^{\infty}-\varepsilon_{1}\right) \lambda^{2}(\rho N)^{-1}\|x\|_{\Omega}\left(\int_{0}^{1} \varphi(s) c_{1}(s) d s\right) \cdot u^{*} \\
& =\left(h_{1}^{\infty}-\varepsilon_{1}\right) \frac{\lambda^{2}}{\rho N^{2}}\left(\int_{0}^{1} \varphi(s) c_{1}(s) d s\left\|u^{*}\right\|\right) \cdot \frac{N\|x\|_{\Omega}}{\left\|u^{*}\right\|} u^{*} \\
& \geq \frac{N\|x\|_{\Omega}}{\left\|u^{*}\right\|} \cdot u^{*}
\end{aligned}
$$

and consequently,

$$
\|T x\|_{\Omega} \geq\|x\|_{\Omega}, \quad \forall x \in \Omega,\|x\|_{\Omega}=R .
$$

Similarly, by condition (H4), there exists $r_{2}>0$ such that

$$
h_{2}\left(u_{1}, \ldots, u_{n-1}\right) \geq\left(h_{1}^{0}-\varepsilon_{2}\right) \sum_{k=1}^{n-1}\left\|u_{k}\right\|, \quad \forall u_{k} \in P, 0<\sum_{k=1}^{n-1}\left\|u_{k}\right\| \leq r_{2},
$$

where $\varepsilon_{2}>0$ satisfies $\left(h_{1}^{0}-\varepsilon_{2}\right) \nabla \geq 1$.

Therefore,

$$
f\left(t, u_{1}, \ldots, u_{n-1}\right) \geq\left(h_{1}^{0}-\varepsilon_{2}\right) \sum_{k=1}^{n-1}\left\|u_{k}\right\| \cdot c_{2}(t) u_{*}, \quad \forall u_{k} \in P, 0<\sum_{k=1}^{n-1}\left\|u_{k}\right\| \leq r_{2} .
$$

Choose

$$
0<r<\min \left\{\left(\sum_{k=0}^{n-2} \frac{1}{k !}\right)^{-1} r_{2}, \xi\right\}
$$

Then, for $t \in I_{\tau}, x \in \Omega,\|x\|_{\Omega}=r$, similar to (3.10), we have

$$
\begin{aligned}
(T x)(t) & \geq\left(h_{1}^{0}-\varepsilon_{2}\right) \lambda \int_{0}^{1} \varphi(s) c_{2}(s)\|x(s)\| d s \cdot u_{*} \\
& \geq\left(h_{1}^{0}-\varepsilon_{2}\right) \lambda^{2}(\rho N)^{-1}\|x\|_{\Omega}\left(\int_{0}^{1} \varphi(s) c_{2}(s) d s\right) \cdot u_{*} \\
& =\left(h_{1}^{0}-\varepsilon_{2}\right) \frac{\lambda^{2}}{\rho N^{2}}\left(\int_{0}^{1} \varphi(s) c_{2}(s) d s\left\|u_{*}\right\|\right) \cdot \frac{N\|x\|_{\Omega}}{\left\|u_{*}\right\|} u_{*} \\
& \geq \frac{N\|x\|_{\Omega}}{\left\|u_{*}\right\|} \cdot u_{*}
\end{aligned}
$$


which implies

$$
\|T x\|_{\Omega} \geq\|x(s)\|_{\Omega}, \quad \forall x \in \Omega,\|x\|_{\Omega}=r .
$$

On the other hand, according to Lemma 3.3 and (3.6), we get

$$
(T x)(t) \leq \rho \int_{0}^{1} \varphi(s) f\left(s, I_{0^{+}}^{n-2} x(s), \ldots, I_{0^{+}}^{1} x(s), x(s)\right) d s .
$$

By condition (H1), for $t \in I, x \in \Omega,\|x\|_{\Omega}=\xi$, we have

$$
\left\|f\left(t, u_{1}, \ldots, u_{n-1}\right)\right\| \leq a(t) h\left(\left\|u_{1}\right\|, \ldots,\left\|u_{n-1}\right\|\right) \leq M_{\xi} a(t) .
$$

Therefore,

$$
\|(T x)(t)\|_{\Omega} \leq \rho N M_{\xi} \cdot \int_{0}^{1} \varphi(s) a(s) d s<\xi=\|x\|_{\Omega} .
$$

Applying Lemma 2.7 to (3.12), (3.13) and (3.14) yields that $T$ has a fixed point $x_{1} \in \bar{\Omega}_{r, \xi}$, $r \leq\left\|x_{1}\right\| \leq \xi$, and a fixed point $x_{2} \in \bar{\Omega}_{\xi, R}, \xi \leq\left\|x_{2}\right\| \leq R$. Noticing (3.13), we get $\left\|x_{1}\right\| \neq \xi$ and $\left\|x_{2}\right\| \neq \xi$. This and Lemma 3.4 complete the proof.

Theorem 3.7 Let the cone P be normal and conditions $(\mathrm{H} 0) \sim(\mathrm{H} 3)$ hold. In addition, assume that the following condition is satisfied:

(H6)

$$
\frac{h\left(\left\|u_{1}\right\|, \ldots,\left\|u_{n-1}\right\|\right)}{\sum_{k=1}^{n-1}\left\|u_{k}\right\|} \rightarrow 0, \quad \text { as } u_{k} \in P, \sum_{k=1}^{n-1}\left\|u_{k}\right\| \rightarrow 0^{+}
$$

Then problem (1.1) has at least one positive solution.

Proof By (H3), we can choose $R>N \rho \lambda^{-1} r_{1}$. As in the proof of Theorem 3.6, it is easy to see that (3.11) holds. On the other hand, considering (3.15), there exists $r_{3}>0$ such that

$$
h\left(\left\|u_{1}\right\|, \ldots,\left\|u_{n-1}\right\|\right) \leq \varepsilon_{3} \sum_{k=1}^{n-1}\left\|u_{k}\right\|, \quad \text { for } t \in I, u_{k} \in P, 0<\sum_{k=1}^{n-1}\left\|u_{k}\right\| \leq r_{3},
$$

where $\varepsilon_{3}>0$ satisfies

$$
\varepsilon_{3}=\left(N \rho \sum_{k=1}^{n-1} \frac{1}{k !} \int_{0}^{1} \varphi(s) a(s) d s\right)^{-1} .
$$

Choose $0<r^{*}<\min \left\{\left(\sum_{k=0}^{n-2} \frac{1}{k !}\right)^{-1} r_{3}, R\right\}$. For $t \in I, x \in \Omega,\|x\|_{\Omega}=r^{*}$, it follows from (3.5) that

$$
\begin{aligned}
& 0<(\rho N)^{-1} \lambda r^{*} \leq\|x\|<r_{3}, \\
& 0<(\rho N)^{-1} \lambda r^{*} \leq \sum_{k=1}^{n-1}\left\|I_{0^{+}}^{n-1-k} x(t)\right\| \leq \sum_{k=1}^{n-1} \frac{1}{k !}\|x\|<r_{3} .
\end{aligned}
$$


Then, for $t \in I, x \in \Omega,\|x\|_{\Omega}=r^{*}$, we have

$$
\begin{aligned}
\|(T x)(t)\| & \leq \rho \int_{0}^{1} \varphi(s)\left\|f\left(s, I_{0^{+}}^{n-2} x(s), \ldots, I_{0^{+}}^{1} x(s), x(s)\right)\right\| d s \\
& \leq \rho \int_{0}^{1} \varphi(s) a(s) h\left(\left\|I_{0^{+}}^{n-2} x(s)\right\|, \ldots,\left\|I_{0^{+}}^{1} x(s)\right\|,\|x(s)\|\right) d s \\
& \leq \varepsilon_{3} \rho \int_{0}^{1} \varphi(s) a(s)\left(\sum_{k=1}^{n-2}\left\|I_{0^{+}}^{n-1-k} x(s)\right\|+\|x(s)\|\right) d s \\
& \leq N \varepsilon_{3} \rho \sum_{k=0}^{n-1} \frac{1}{k !} r^{*} \int_{0}^{1} \varphi(s) a(s) d s=r^{*},
\end{aligned}
$$

and consequently,

$$
\|(T x)(t)\|_{\Omega} \leq\|x\|_{\Omega}, \quad \forall x \in \Omega,\|x\| \leq r^{*}
$$

Since $0<r^{*}<R$, applying Lemma 2.7 to (3.11) and (3.17) yields that $T$ has a fixed point $x_{0} \in \bar{\Omega}_{r^{*}, R}, r^{*} \leq\left\|x_{0}\right\| \leq R$. This and Lemma 3.5 complete the proof.

\section{An example}

Consider the following system of scalar differential equations of fractional order:

$$
\left\{\begin{aligned}
&-D^{\frac{5}{2}} u_{k}(t)= \frac{1}{24} u_{k}(t)+\frac{1+t}{324 k^{2}}\left\{\left[2 u_{k+2}(t)+2 u_{k+1}^{\prime}(t)+\sum_{j=1}^{\infty} u_{j}(t)+\sum_{j=1}^{\infty} u_{2 j}^{\prime}(t)\right]^{3}\right. \\
&\left.+\sqrt{3 u_{2 k}(t)+3 u_{k+1}^{\prime}(t)+\sum_{j=1}^{\infty} u_{j}(t)+\sum_{j=1}^{\infty} u_{j}^{\prime}(t)}\right\}, \quad t \in I, \\
& u_{k}(0)=u_{k}^{\prime}(0)=0, \quad \frac{1}{4} u_{k}(0)-\frac{1}{2} u_{k}^{\prime}(0)=0, \\
& u_{k}(1)+\frac{1}{2} u_{k}^{\prime}(1)=0, \quad k=1,2,3, \ldots
\end{aligned}\right.
$$

Conclusion Problem (4.1) has at least two positive solutions.

Proof Let $E=l^{1}=\left\{u=\left(u_{1}, u_{2}, \ldots, u_{k}, \ldots\right): \sum_{k=1}^{\infty}\left|u_{k}\right|<\infty\right\}$ with the norm $\|u\|=\sum_{k=1}^{\infty}\left|u_{k}\right|$, and $P=\left\{\left(u_{1}, \ldots, u_{k}, \ldots\right): u_{k} \geq 0, k=1,2,3, \ldots\right\}$. Then $P$ is a normal cone in $E$ with a normal constant $N=1$, and system (4.1) can be regarded as a boundary value problem of the form (1.1). In this situation, $q=\frac{5}{2}, n=3, \alpha=\frac{1}{4}, \beta=\frac{1}{2}, \gamma=1, \delta=\frac{1}{2}, u=\left(u_{1}, \ldots, u_{n}, \ldots\right)$, $f=\left(f_{1}, f_{2}, \ldots, f_{n}, \ldots\right)$, in which

$$
\begin{aligned}
f_{k}\left(t, u_{k}, u_{k}^{\prime}\right)= & \frac{1}{24} u_{k}(t)+\frac{1+t}{324 k^{2}}\left\{\left[2 u_{k+2}(t)+2 u_{k+1}^{\prime}(t)+\sum_{j=1}^{\infty} u_{j}(t)+\sum_{j=1}^{\infty} u_{2 j}^{\prime}(t)\right]^{3}\right. \\
& +\sqrt{\left.3 u_{2 k}(t)+3 u_{k+1}^{\prime}(t)+\sum_{j=1}^{\infty} u_{j}(t)+\sum_{j=1}^{\infty} u_{j}^{\prime}(t)\right\}} .
\end{aligned}
$$

By calculating, we have $\rho=\frac{4}{5}, \lambda \approx 0.1515$, and

$$
\frac{1}{2}=\beta>\frac{n-q}{q-n+1} \alpha=\frac{1}{4},
$$


and

$$
\rho^{*}=\rho \int_{0}^{1} \varphi(s) d s=\frac{1}{5 \sqrt{\pi}} \int_{0}^{1}(2+s)\left(\sqrt{1-s}+\frac{1}{4 \sqrt{1-s}}\right) d s=\frac{44}{75 \sqrt{\pi}} \approx 0.3310,
$$

which implies that condition (H0) is satisfied. Observing the inequality $\sum_{k=1}^{\infty} \frac{1}{k^{2}}<2$, we get, by (4.2),

$$
\|f(t, u, v)\|=\sum_{k=1}^{\infty}\left|f_{k}\left(t, u_{k}, v_{k}\right)\right| \leq \frac{1+t}{3}\left(\frac{1}{8}\|u\|+\frac{1}{2}(\|u\|+\|v\|)^{3}+\frac{1}{27} \sqrt{\|u\|+\|v\|}\right) .
$$

Hence $(\mathrm{H} 1)$ is satisfied for $a(t)=\frac{1+t}{3}$, and

$$
h(x, y)=\frac{1}{8} x+\frac{1}{2}(x+y)^{3}+\frac{1}{27} \sqrt{x+y} .
$$

Now, we check condition (H2). Obviously, $f: I \times P_{r}^{2} \rightarrow P$ for any $r>0$, and $f$ is uniformly continuous on $I \times P_{r}^{2}$. Let $f=f^{(1)}+f^{(2)}$, where $f^{(1)}=\left(f_{1}^{(1)}, \ldots, f_{k}^{(1)}, \ldots\right)$ and $f^{(2)}=$ $\left(f_{1}^{(2)}, \ldots, f_{k}^{(2)}, \ldots\right)$, in which

$$
\begin{aligned}
f_{k}^{(1)}(t, u, v)= & \frac{1+t}{324 k^{2}}\left\{\left[2 u_{k+2}(t)+2 u_{k+1}^{\prime}(t)+\sum_{j=1}^{\infty} u_{j}(t)+\sum_{j=1}^{\infty} u_{2 j}^{\prime}(t)\right]^{3}\right. \\
& +\sqrt{\left.3 u_{2 k}(t)+3 u_{k+1}^{\prime}(t)+\sum_{j=1}^{\infty} u_{j}(t)+\sum_{j=1}^{\infty} u_{j}^{\prime}(t)\right\} \quad(k=1,2,3, \ldots),}
\end{aligned}
$$

and

$$
f_{k}^{(2)}(t, u, v)=\frac{1}{24} u_{k}(t) \quad(k=1,2,3, \ldots) .
$$

For any $t \in I$ and bounded subsets $D_{1}, D_{2} \subset E$, by (4.4), (4.5), we know

$$
\alpha\left(f^{(2)}\left(I, D_{1}, D_{2}\right)\right) \leq \frac{1}{24} \alpha\left(D_{1}\right), \quad \forall t \in I, D_{1}, D_{2} \subset E,
$$

and

$$
\begin{aligned}
0 & \leq\left\|f^{(1)}(t, u, v)\right\|=\sum_{k=1}^{\infty}\left|f_{k}^{(1)}\left(t, u_{k}, v_{k}\right)\right| \\
& \leq(\|u\|+\|v\|)^{3}+\frac{2}{27} \sqrt{\|u\|+\|v\|}, \quad \forall t \in I, u, v \in E .
\end{aligned}
$$

Similar to the proof of [25, Example 2.12], we have

$$
\alpha\left(f^{(1)}\left(t, D_{1}, D_{2}\right)\right)=0, \quad \forall t \in I \text {, bounded sets } D_{1}, D_{2} \subset E \text {. }
$$

It follows from (4.6) and (4.7) that

$$
\alpha\left(f\left(I, D_{1}, D_{2}\right)\right) \leq \frac{1}{24} \alpha\left(D_{1}\right), \quad \forall t \in I, D_{1}, D_{2} \subset E,
$$


and

$$
\frac{2}{\rho^{*}}\left(\sum_{k=1}^{n-2} \frac{L_{k}}{(n-2-k) !}+L_{n-1}\right)=\frac{24}{29 \sqrt{\pi}} \approx 0.2518<1,
$$

i.e., condition (H2) holds for $L_{1}=\frac{1}{24}, L_{2}=0$.

On the other hand, by (4.2), we have

$$
f_{k}(t, u, v) \geq \frac{1+t}{324 k^{2}}(\|u\|+\|v\|)^{3}, \quad \forall t \in I_{\tau}, u, v \in P(k=1,2,3, \ldots),
$$

and

$$
f_{k}(t, u, v) \geq \frac{1+t}{324 k^{2}} \sqrt{\|u\|+\|v\|}, \quad \forall t \in I_{\tau}, u, v \in P(k=1,2,3, \ldots) .
$$

Hence condition (H3) is satisfied for

$$
c_{1}(t)=\frac{1+t}{324 k^{2}}, \quad h_{1, k}(u, v)=(\|u\|+\|v\|)^{3}, \quad \text { and } \quad u^{*}=\left(1, \ldots, \frac{1}{k^{2}}, \ldots\right)
$$

in this situation,

$$
h_{1, k}^{\infty}=\lim _{\|u\|+\|v\| \rightarrow \infty} \frac{(\|u\|+\|v\|)^{3}}{\|u\|+\|v\|}=\infty>\Delta^{-1} .
$$

And condition ( $\mathrm{H} 4)$ is also satisfied for

$$
c_{2}(t)=\frac{1+t}{324 k^{2}}, \quad h_{2, k}(u, v)=\sqrt{\|u\|+\|v\|}, \quad \text { and } \quad u_{*}=\left(1, \ldots, \frac{1}{k^{2}}, \ldots\right) \text {, }
$$

in this situation,

$$
h_{2, k}^{\infty}=\lim _{\|u\|+\|v\| \rightarrow 0} \frac{\sqrt{\|u\|+\|v\|}}{\|u\|+\|v\|}=\infty>\nabla^{-1}
$$

Finally, choose $\xi=1$. It is easy to check that condition (H5) is satisfied. In this case, $M_{\xi} \approx 4.1774$, and so

$$
\rho N M_{\xi} \int_{0}^{1} \varphi(s) a(s) d s \approx 0.9604<\xi=1 .
$$

From Theorem 3.6, the conclusion follows and the proof is complete. 


\section{Acknowledgements}

The authors are highly grateful for the referees's careful reading and comments on this paper. The first author is supported financially by Hunan Provincial Natural Science Foundation of China (Grant No: 13JJ3106).

Received: 9 July 2013 Accepted: 29 October 2013 Published: 27 Nov 2013

\section{References}

1. Podlubny, I: Fractional Differential Equations. Academic Press, San Diego (1999)

2. Kilbas, AA, Srivastava, HM, Trujillo, JJ: Theory and Applications of Fractional Differential Equations. North-Holland Mathematics Studies, vol. 204. Elsevier, Amsterdam (2006)

3. Sabatier, J, Agrawal, OP, Machado, JAT (eds.): Advances in Fractional Calculus: Theoretical Developments and Applications in Physics and Engineering. Springer, Dordrecht (2007)

4. Baleanu, D, Diethelm, K, Scalas, E, Trujillo, Jj: Fractional Calculus Models and Numerical Methods. Series on Complexity, Nonlinearity and Chaos. World Scientific, Boston (2012)

5. Lakshmikantham, V: Theory of fractional functional differential equations. Nonlinear Anal. 69, 337-3343 (2008)

6. Zhang, S: Positive solutions to singular boundary value problem for nonlinear fractional differential equation. Comput. Math. Appl. 59, 1300-1309 (2010)

7. Bai, Z: On positive solutions of a nonlocal fractional boundary value problem. Nonlinear Anal. 72, 916-924 (2010)

8. Jankowski, T: Initial value problems for neutral fractional differential equations involving a Riemann-Liouville derivative. Appl. Math. Comput. 219, 7772-7776 (2013)

9. Goodrich, CS: Existence of a positive solution to a class of fractional differential equations. Appl. Math. Lett. 23, 1050-1055 (2010)

10. Zhao, Y, Chen, H, Huang, L: Existence of positive solutions for nonlinear fractional functional differential equation. Comput. Math. Appl. 64, 3456-3467 (2012)

11. Zhang, $X, L i u, L, W u, Y:$ Multiple positive solutions of a singular fractional differential equation with negatively perturbed term. Math. Comput. Model. 55, 1263-1274 (2012)

12. Wang, G: Monotone iterative technique for boundary value problems of a nonlinear fractional differential equations with deviating arguments. J. Comput. Appl. Math. 236, 2425-2430 (2012)

13. Wang, G, Baleanu, D, Zhang, L: Monotone iterative method for a class of nonlinear fractional differential equations. Fract. Calc. Appl. Anal. 15, 244-252 (2012)

14. Zhao, Y, Ye, G, Chen, H: Multiple positive solutions of a singular semipositone integral boundary value problem for fractional q-derivatives equation. Abstr. Appl. Anal. 2013, Article ID 643571 (2013)

15. El-Shahed, M, Nieto, JJ: Nontrivial solutions for a nonlinear multi-point boundary value problem of fractional order. Comput. Math. Appl. 59, 3438-3443 (2010)

16. Zhao, Y, Chen, $\mathrm{H}$, Zhang, Q: Existence results for fractional q-difference equations with nonlocal $q$-integral boundary conditions. Adv. Differ. Equ. 2013, 48 (2013)

17. Ahmad, B: Existence of solutions for irregular boundary value problems of nonlinear fractional differential equations. Appl. Math. Lett. 23, 390-394 (2010)

18. Wang, J, Zhou, Y, Fečkan, M: Abstract Cauchy problem for fractional differential equations. Nonlinear Dyn. 71, 685-700 (2013)

19. Salem, AH: On the fractional order $m$-point boundary value problem in reflexive Banach spaces and weak topologies J. Comput. Appl. Math. 224, 565-572 (2009)

20. Balachandran, $\mathrm{K}$, Trujillo, JJ: The nonlocal Cauchy problem for nonlinear fractional integrodifferential equations in Banach spaces. Nonlinear Anal. 72, 4587-4593 (2010)

21. Balachandran, K, Kiruthika, S, Trujillo, JJ: Existence results for fractional impulsive integrodifferential equations in Banach spaces. Commun. Nonlinear Sci. Numer. Simul. 16, 1970-1977 (2011)

22. Lv, Z, Liang, J, Xiao, T: Solutions to the Cauchy problem for differential equations in Banach spaces with fractional order. Comput. Math. Appl. 62, 1303-1311 (2011)

23. Su, X: Solutions to boundary value problem of fractional order on unbounded domains in a Banach space. Nonlinear Anal. 74, 2844-2852 (2011)

24. Zhang, L, Ahmad, B, Wang, G, Agarwal, RP: Nonlinear fractional integro-differential equations on unbounded domains in a Banach space. J. Comput. Appl. Math. 249, 51-56 (2013)

25. Guo, D, Lakshmikantham, V: Nonlinear Problems in Abstract Cones. Academic Press, New York (1988)

10.1186/1687-1847-2013-344

Cite this article as: Zhao et al.: Positive solutions to boundary value problems of a high-order fractional differential equation in a Banach space. Advances in Difference Equations 2013, 2013:344 